\title{
An Investigation into Alternative Methods for the Defuzzification of an Interval Type-2 Fuzzy Set
}

\author{
Simon Coupland, Member, IEEE, and Robert John, Member, IEEE
}

\begin{abstract}
The number of applications of interval type-2 fuzzy logic to real world problems is growing. To date such systems have used type-reduction or an approximation of typereduction to arrive at final crisp output from the system. This paper describes the novel direct defuzzifier for interval type-2 fuzzy sets. A number of fuzzy systems are compared with direct defuzzifier. We compare each defuzzifiers output surface in a simple rule-based system. The direct defuzzifier compares favourably with the type-reduction method under the minimum t-norm. Also, we found that the non-stationary approach provided interesting results.
\end{abstract}

\section{INTRODUCTION}

Recent years have seen a large growth in the number of reported applications of interval type- 2 fuzzy logic. These systems include mobile robot navigation[8], process control[15], engine control[11] and object tracking[5]. Interval type-2 fuzzy logic is variation of general type-2 fuzzy logic.

A type-2 fuzzy set maps elements of the domain $X$ to type1 fuzzy numbers whose domain $J$ is bounded the interval $[0,1]$ i.e.

$$
\tilde{A}=\left\{\left((x, u), \mu_{\tilde{A}}(x, u)\right), \forall x \in X, \forall u \in J_{x} \subseteq[0,1]\right\}
$$

where $\mu_{\tilde{A}}(x, u)$ is in the interval $[0,1]$. Interval type-2 fuzzy sets constrain general type- 2 fuzzy sets by only allowing $\mu_{\tilde{A}}(x, u)$ to take a value of zero or one i.e.

$$
\tilde{A}=\left\{\left((x, u), \mu_{\tilde{A}}(x, u)\right), \forall x \in X, \forall u \in J_{x} \subseteq[0,1]\right\}
$$

where $J$ is in the interval $[0,1], \mu_{\tilde{A}}(x, u)$ is an element of the crisp set $\{0,1\}$. An example interval type-2 fuzzy set $\tilde{F}$ is depicted in Figure 1. $\tilde{F}$ is a triangular fuzzy set with an uncertain centre point. Interval type-2 fuzzy sets

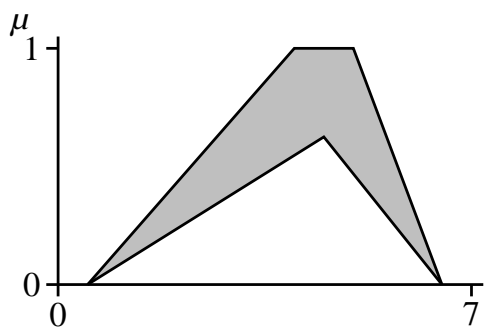

Fig. 1. The Interval Type-2 Fuzzy Set $\tilde{F}$.

are three dimensional, however since every point in the third dimension is either at zero or one it is common for such set to be depicted in two dimension as with Figure 1.

Simon Coupland and Robert John are with the Centre for Computational Intelligence at De Montfort University, The Gateway, Leicester, LE1 9BH, United Kingdom. E-mail simon@dmu.ac.uk and rij@dmu.ac.uk
Furthermore it is quite often possible to represent an interval type-2 fuzzy set with two type-1 fuzzy sets representing the upper and lower bounds of the interval type-2 fuzzy set. This efficient representation coupled with the efficient type-reduction strategies are the factors that have driven the application of this technology to real world problems. General type-2 fuzzy logic, despite recent advances, is yet achieve the low computational expense of interval systems and as such has not seen a similar growth in applications.

Most applied interval type-2 fuzzy system rely on typereduction or an approximation of the type-reduction procedure to arrive at a crisp output. We believe that some of the characteristics of type-reduction may make it unsuitable for some applications. In this paper we therefore propose an alternative to type-reduction, the direct defuzzifier, which is adapted from geometric fuzzy logic [1], [2], [3], [4] and is both computationally efficient and the level of computation is known beforhand. Furthermore this paper reports the first use of the sampling defuzzifier [7] with interval type-2 fuzzy systems. We also use the non-stationary fuzzy systems approach [6], [14] to emulate the performance of interval type-2 fuzzy systems. An experiment is designed which compares the characteristics of these systems with a type-1 fuzzy system and an interval type- 2 system using type-reduction. The results of this experiment are, we believe, interesting and raise some important question for practitioners in the field of type-2 fuzzy logic.

The rest of this paper is organised as follows: the following section describes centroid type-reduction, section III describes the direct defuzzifier, section IV the sampling defuzzifier, section $\mathrm{V}$ describes the non-stationary approach, section VI outlines the methods that will be used to investigate the various systems, section VII gives the results of that investigation, section VIII discussion the outcomes of the investigation and section IX draws conclusions from this work.

\section{TYPE-REDUCTION}

Type-reduction [9], [10], [12] is an extension of the type-1 defuzzification procedure for type- 2 fuzzy sets. Throughout his paper we only consider the centroid defuzzifier. This is because the direct defuzzier presented in the following section relates to geometric fuzzy logic. The only defuzzifier currently available in geometric fuzzy logic is the centroid or centre of area defuzzifier. Type-reduction calculates a set that is a distribution of the possible centroids of that set. In the case of an interval type-2 fuzzy set $\tilde{B}$, type-reduction yields an interval set. The end points of this set give the 
range of possible values that the centroid of $\tilde{B}$ could take. The defuzzified value is given by the midpoint of these end points. Mendel [12] defined the centroid of an interval type-2 fuzzy set $\tilde{A}$ as:

$$
C_{\tilde{A}}=\int_{\theta_{1} \in J_{x_{1}}} \ldots \int_{\theta_{N} \in J_{x_{N}}} 1 / \frac{\sum_{i=1}^{N} x_{i} \theta_{i}}{\sum_{i=1}^{N} \theta_{i}}=\left[C_{l}, C_{r}\right]
$$

Which can be expressed by the algorithm given below:

1) Enumerate all possible embedded sets in $\tilde{A}$.

2) Take the centroid $C$ of each of these embedded sets.

3) Let the minimum value of $C$ be the lower endpoint $C_{l}$.

4) Let the maximum value of $C$ be the upper endpoint $C_{r}$.

5) Let the type-reduced set $=\left[C_{l}, C_{r}\right]$.

Where an embedded interval type- 2 fuzzy set $A_{e}$ is defined as

$$
A_{e}=\sum_{i=1}^{N}\left[1 / u_{i}\right] / x_{i}, u_{i} \in J_{x_{i}}, J \subseteq[0,1]
$$

which follows from the definition of an embedded type-2 fuzzy set given by Mendel and John [13].

The full type-reduction algorithm is rarely used in real systems. This is because more computationally efficient methods have been devised, one of which is the Karnik and Mendel iterative procedure [10]. Karnik and Mendel discovered that the embedded sets that give the endpoints of the type-reduced interval are always of a certain form. The membership function of the embedded set that gives the lower endpoint will always follow the upper bound of the set to a switch point and will then follow the lower bound. Conversely the membership function of the embedded set that gives the upper endpoint will always follow the lower bound of the set to a switch point and will then follow upper bound. Figure 2 (a) depicts the embedded set $\tilde{F}_{e}^{C_{l}}$ that gives (a)

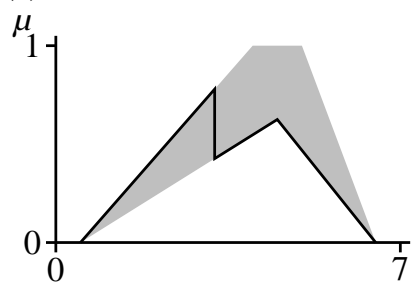

(b)

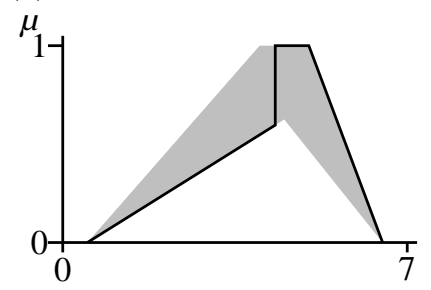

Fig. 2. (a) The Embedded Set $\tilde{F}_{e}^{C_{r}}$. (b) The Embedded Set $\tilde{F}_{e}$.

left endpoint $C_{l}$ of the type-reduced set of $\tilde{F}$ depicted in Figure 1. Figure 2 (b) depicts the embedded set $\tilde{F}_{e} \widetilde{C}_{r}$ that gives right endpoint $C_{r}$ of the type-reduced set of $\tilde{F}$ depicted in Figure 1.

As mentioned earlier we feel that type-reduction may be unsuitable for some applications. Three aspects of typereduction are of concern, which will be explored in turn.

1) The type-reduced set is defined by only two embedded sets.

2) These embedded sets rarely resemble the shape of the overall set.

3) The computational expense of the iterative method is unpredictable.
The first point is of concern as it separates type-reduction of a general type- 2 fuzzy set from an interval type- 2 fuzzy set. Type-reduction of a general type-2 fuzzy set is computationally expensive. The centroid value of each of the embedded sets is used to arrive at type-1 fuzzy set. All the information contained in the type- 2 fuzzy set is used to arrive at an output. Conversely when type-reducing interval type2 fuzzy sets a great deal of the information contain in the fuzzy set is never considered when calculating an output.

The second point concerns the membership functions of the embedded sets identified by type-reduction. Consider Figure 1 which depicts an interval type- 2 fuzzy set. This set has a triangular membership function where there is some uncertainty about the midpoint. Now consider Figures 2 (a) and (b), the embedded sets in that triangular membership function identified by type-reduction. The membership functions of these two embedded set do not resemble the original triangular membership function. From a technical point of view this is not an issue, however when we think of fuzzy sets as linguistic variables problems may arise with the question:

What name do we give the embedded sets in Figure 2?

To paraphrase this question, what do these embedded sets mean to a lay person? The result of interval type- 2 system is defined by these two embedded sets, as such it seems sensible that lay people should be able to interpret them.

The third point has to some extent already been overcome by other work. Although low and bounded, the exact computational expense of the iterative method is unpredictable. Approximate methods such as the Wu-Mendel uncertainty bounds method [16] already provide static levels of computation at the price of using an approximation to the result.

This section has described type-reduction of an interval type-2 fuzzy set. Some potential problems with typereduction have been identified. These are the points that the novel direct defuzzifier presented in the following section seeks to address.

\section{THE DIRECT DEFUZZIFIER}

In [1], [2], [3], [4] the Authors introduced a complete geometric model of type- 1 and interval type- 2 fuzzy logic. The direct defuzzifier is the discrete equivalent of the geometric defuzzifier used in geometric fuzzy logic. Geometric fuzzy logic involves replacing the discrete computational model of fuzzy sets with a continuous computational model based on geometric primitives. All operations on geometric fuzzy sets are geometric manipulations of the primitives that make up the geometric fuzzy sets. Type-reduction relies on the discrete model of fuzzy sets for practical implementation. The iterative method achieves such a high performance by effectively performing an heuristic search through these discrete points. Were the sets to be continuous this search would be infinite. No geometric equivalent to type-reduction is available for geometric fuzzy sets as they are defined over continuous domains. Instead geometric fuzzy sets use the geometric centre of area to find the centroid of a fuzzy set. To date no other defuzzication methods other than the centre of 


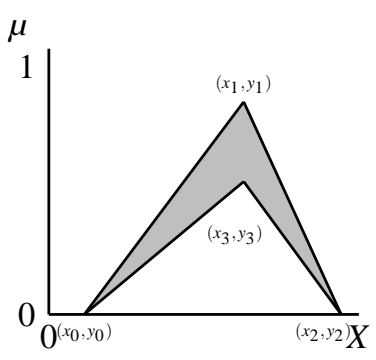

Fig. 3. The Polygon $P_{\tilde{A}}$.

area have been defined for geometric fuzzy sets. Both type-1 and interval type-2 fuzzy sets are defined as 2-dimensional polygons consisting of a number of ordered 2-dimensional vertices. The centre of area of such fuzzy sets is defined as the centre of area of this polygon.

Let the geometric interval type-2 fuzzy set $\tilde{F}$ consist of upper and lower membership functions denoted by $\mu_{\tilde{F}}$ and $\mu_{\tilde{F}}$ respectively. Furthermore the upper membership function of $\tilde{F}$ consist of a number of vertices such that $\mu_{\tilde{\tilde{F}}}=\overline{\left(x_{0}, y_{0}\right)}, \overline{\left(x_{1}, y_{1}\right)}, \ldots, \overline{\left(x_{n}, y_{n}\right)}$. The lower membership function of $\tilde{F}$ also consists of a number vertices, namely $\mu_{\underline{\tilde{F}}}=\left(x_{0}, y_{0}\right),\left(x_{1}, y_{1}\right), \ldots,\left(x_{n}, y_{n}\right)$. These two geometric membership functions are then joined together in order to form the closed non-intersecting polygon $P_{\tilde{F}}$ such that $P_{\tilde{F}}=$ $\overline{\left(x_{0}, y_{0}\right)}, \overline{\left(x_{1}, y_{1}\right)}, \ldots, \overline{\left(x_{n}, y_{n}\right)},\left(x_{n}, y_{n}\right), \ldots,\left(x_{1}, y_{1}\right),\left(x_{0}, y_{0}\right)$. For simplicity that the indices of $P_{\tilde{F}}$ be renumbered, $P_{\tilde{F}}=$ $\left(x_{0}, y_{0}\right),\left(x_{1}, y_{1}\right), \ldots,\left(x_{n}, y_{n}\right)$. The $x$ component of the centre of area of $P_{\tilde{F}}$ and therefore the centroid of $\tilde{F}$ is given by equation 5 .

$$
C_{x}=\frac{\sum_{i=0}^{n}\left(x_{i}+x_{i+1}\right)\left(x_{i} y_{i+1}-x_{i+1} y_{i}\right)}{3\left(\sum_{i=0}^{n} x_{i} y_{i+1}-x_{i+1} y_{i}\right)}
$$

This method essentially decomposes the polygon $P_{\tilde{F}}$ into a collection of triangles. The centroid value is the weighted average of the centre and area of these triangles. Consider the example polygon $P_{\tilde{A}}$ depicted in Figure 3 . This polygon can be deconstructed in the four triangles $t_{0}$ to $t_{3}$ depicted in Figures 4 (a) to (d).

Geometric fuzzy sets need to be deconstructed into primitive objects for defuzzification. Discrete fuzzy sets are already a simple collection of discrete points and therefore no equivalent deconstruction is required. In order to find the geometric centre of area of an interval type- 2 fuzzy we therefore only need to find the weighted average of the area and centroid of each of the discrete points. Since each point covers the same area over the domain, the $x$-dimension we can take the area of each point as height and the centroid as the points domain value. Therefore the discrete equivalent of the geometric defuzzifier, which we call the direct defuzzifier, (a)

(b)

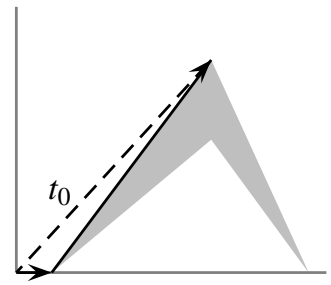

(c)

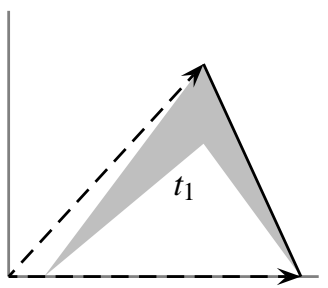

(d)
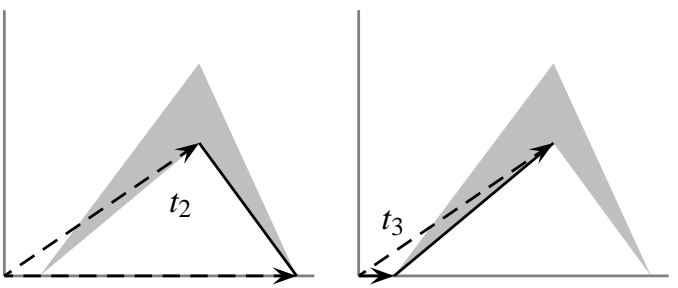

Fig. 4. The Triangles $t_{0}, t_{1}, t_{2}$ and $t_{3}$.

can therefore be given by equation 6 .

$$
\operatorname{COA}_{\tilde{F}}=\frac{\sum_{i=0}^{m} \mu_{\tilde{\tilde{F}}}\left(x_{i}\right) x_{i}+\sum_{i=0}^{n} \mu_{\tilde{\tilde{F}}}\left(x_{i}\right) x_{i}}{\sum_{i=0}^{m} \mu_{\tilde{\tilde{F}}}\left(x_{i}\right)+\sum_{i=0}^{n} \mu_{\tilde{\tilde{F}}}\left(x_{i}\right)}
$$

Where $\mu_{\tilde{F}}$ and $\mu_{\tilde{F}}$ are the upper and lower membership functions of the interval type-2 fuzzy set $\tilde{F}$ respectively. Both $\mu_{\tilde{F}}$ and $\mu_{\tilde{F}}$ are over the discrete domain $X$ and consist of $m$ and $n$ points respectively.

The direct defuzzifier is consistent with Mendel's design principle [12]. This states that the design of type-2 systems should be guided by the principle that when uncertainty disappears the type- 2 systems are equal to the type- 1 systems. This can be demonstrated for the direct defuzzifier. When uncertainty disappears in an interval system the upper and lower membership functions become equal. Let the equivalent type- 1 fuzzy set be $F$ with a membership function $\mu_{F}$ of $n$ points be equal to both the upper and lower membership functions of $\tilde{F}$. The centre of area of $F$ is equal to the direct defuzzification of $\tilde{F}$ as shown in equation 7 where $\mu_{F}(x)=\mu_{\tilde{\tilde{F}}}(x)=\mu_{\tilde{F}}(x) \forall x \in X$.

$$
\frac{\sum_{i=0}^{m} \mu_{\tilde{\tilde{F}}}\left(x_{i}\right) x_{i}+\sum_{i=0}^{n} \mu_{\underline{\underline{F}}}\left(x_{i}\right) x_{i}}{\sum_{i=0}^{m} \mu_{\tilde{\tilde{F}}}\left(x_{i}\right)+\sum_{i=0}^{n} \mu_{\underline{\tilde{F}}}\left(x_{i}\right)}=\frac{2 \sum_{i=0}^{n} \mu_{F}\left(x_{i}\right) x_{i}}{2 \sum_{i=0}^{n} \mu_{F}\left(x_{i}\right)}
$$

In this section the novel direct defuzzifier has been defined. This method was developed from geometric fuzzy logic where type-reduction is not possible. This method addresses the concerns with type-reduction as the entire form of the fuzzy is used and computational expense is predictable. The following section presents a second alternative to typereduction, the sampling defuzzifier.

\section{The SAMPling DefuZZIFIER}

The sampling method of defuzzification was first introduced by Greenfield et al in [7]. The technique was developed for general type-2 fuzzy sets to allow such sets 
to be type-reduced within a reasonable time frame. Typereduction finds the centroid by taking the average centroid of all the possible embedded sets in the type- 2 set. The sampling method chooses a number of the embedded sets at random ${ }^{1}$ and uses this randomly chosen subset for type-reduction. The full type-reduction procedure is then performed on this subset of embedded sets. The algorithm for the sampling defuzzifier for an interval type- 2 fuzzy set $\tilde{F}$ is given below.

1) Randomly generate a subset of type- 2 embedded sets of predetermined cardinality from $\tilde{F}$.

2) Calculate the centroid of each of the generated embedded sets.

3) Let the minimum of these centroids be $L$.

4) Let the maximum of these centroid be $R$.

5) Let the final defuzzified value $C=\frac{L+R}{2}$

The sampling method has not yet been used on interval type-2 fuzzy sets. This is largely due to the efficiency of the iterative method which reduces the computational burden of the procedure. The iterative method only requires knowledge of the upper and lower bounds of the interval type-2 fuzzy set where as the sampling method requires discrete secondary membership functions.

This section has given the sampling defuzzifier procedure for an interval type-2 fuzzy logic system. This is the first use of such a defuzzifier on interval rather than general type-2 fuzzy sets. In investigating such a system our aim is gain some knowledge about the sampling defuzzifier and to provide comparative results with the other defuzzifiers explored in this paper. The next section describes nonstationary fuzzy systems.

\section{NON-STATIONARY FUZZY Systems}

Non-stationary fuzzy logic systems [6] are a collection of non-deterministic fuzzy logic systems [14]. Ozen and Garibaldi proposed non-deterministic fuzzy systems as a way of modelling self-variation of human experts. They proposed that by introducing variations of the fuzzy membership functions in a fuzzy logic system the results from such a system may model variation in human decision making. A non-stationary fuzzy set is a fuzzy set where the membership function of that set is altered by a perturbation function. A non-stationary fuzzy logic system is a fuzzy system which uses such non-stationary fuzzy sets. Garibaldi et al [6] define a non-stationary fuzzy set, denoted $\dot{A}$ as being characterised by a membership function $\mu_{\dot{A}}(x, t)$, where $x \in X$ and $\mu_{\dot{A}}(x, t) \in$ $[0,1]$ and $t$ is a free variable time, which is the time at which the fuzzy set is instantiated, i.e.

$$
\dot{A}=\int_{x \in X} \mu_{\dot{A}}(x, t) / x
$$

The membership functions of non-stationary fuzzy sets are generally parametric. Garibaldi et al give the example of the

\footnotetext{
${ }^{1}$ Both the sampling method and the non-stationary fuzzy systems present in this paper make extensive use of randomly generated numbers during the inferencing process. The method used to generate these numbers was the rand() function from the standard $\mathrm{C}$ library. This function returns a pseudorandom integer which can be bounded and scaled for a specific purpose.
}

Gaussian non-stationary fuzzy set medium, where standard deviation, $\sigma$ is the parameter being altered:

$$
\operatorname{medium}(t)=\int_{1}^{2} e^{-\frac{(x-1.6)^{2}}{\sigma(t)^{2}}} / x
$$

Where $\sigma(t)=\sigma+k f(t), k$ is a constant and $f(t)$ is the perturbation function.

By repeating the inferencing process of a non-stationary fuzzy system a distribution of results can be obtained, which to some extent can be viewed as an emulation of a type-2 fuzzy system. In [6] Garibaldi et al compared the bounds and mean results from non-stationary fuzzy systems using over $30,100,1,000$ and 10,000 inferences with comparable interval type-2 systems using various perturbation functions. No definitive conclusions were reached in as to the performance of non-stationary systems in emulating type- 2 systems. Work presented later in this paper extends knowledge in this field.

This section has given a definition of a non-stationary fuzzy system. Like the direct defuzzifier, non-stationary systems have predictable computation levels and make full use of all the information given by a fuzzy set. The following section presents a comparison of a number alternative fuzzy systems including a non-stationary fuzzy system.

\section{INVESTIGATION}

The previous sections have explored three alternatives to type-reduced interval type-2 fuzzy logic systems, the direct defuzzier, the sampling method and non-stationary fuzzy systems. In this section we compare the output surfaces of type-1, type-reduced interval type-2, direct defuzzified interval type-2, sampled interval type- 2 and non-stationary fuzzy logic systems.

To compare the characteristics of each fuzzy method we created a simple system in each of the five fuzzy methods being investigated. Each of these systems uses the following two rules:

- Rule 1: IF $x_{1}$ is $F_{1}$ THEN $y$ is $G_{1}$

- Rule 2: IF $x_{2}$ is $F_{2}$ THEN $y$ is $G_{2}$

Where $x_{1}$ and $x_{2}$ are inputs, $F_{1}$ and $F_{2}$ are antecedent fuzzy sets, $G_{1}$ and $G_{2}$ are consequent fuzzy sets and $y$ is the output from the system. These Mamdani type rules will be combined with a logical $O R$ operation before defuzzification. In the interval type- 2 systems $F_{1}, F_{2}, G_{1}$ and $G_{2}$ will be replaced with $\tilde{F}_{1}, \tilde{F}_{2}, \tilde{G}_{1}$ and $\tilde{G}_{2}$ and in the non-stationary systems they will be replaced with $\dot{F}_{1}, \dot{F}_{2}, \dot{G}_{1}$ and $\dot{G}_{2}$. To compare the characteristics of the various systems a two-dimensional plot of the output surface of each system will be drawn and assessed.

The output surface plots plot the firing strength of rule 1 the along $y$ axis and plot the firing strength of rule 2 along the $\mathrm{x}$ axis. Each rule will be fired from 0 to 1 at intervals 0.01 , each axis covering 100 data points, each plot describing 10,000 data points. The result at each data point is represented by a tone between black and white where white is 14 and black is 3 . This range covers every possible output from all the systems being compared. Lines will be 


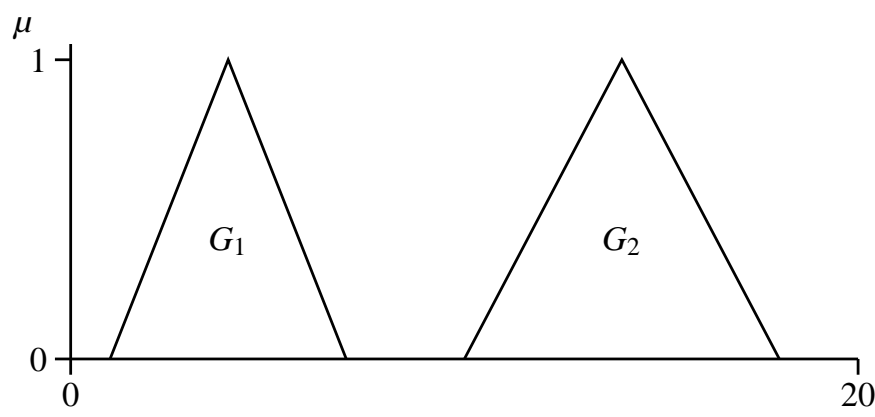

Fig. 5. The Type-1 Fuzzy Sets $G_{1}$ and $G_{2}$.

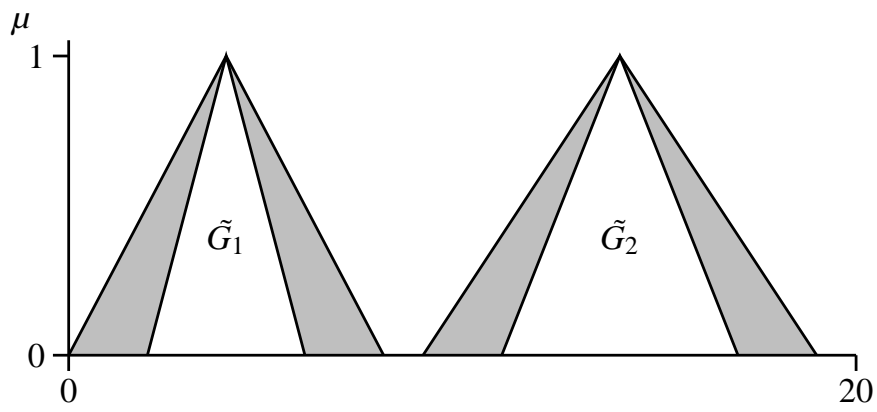

Fig. 6. The Interval Type-2 Fuzzy Sets $\tilde{G}_{1}$ and $\tilde{G_{2}}$.

plotted at every interval of 0.25 between 3 and 14. Plotting these surfaces in this way allows the characteristics of each system to be visualised in 2-dimensions. The steepness and smoothness of each surface can be easily viewed in such plots.

This surface is different from the control surface, which maps inputs to outputs. In this paper we are only interested in the defuzzification characteristics of the various systems. We use the output surface as it is more focused on the defuzzification step. Unlike a control surface the rule antecedents have no impact on an output surface and as such the impact of defuzzifcation will therefore carry greater emphasis. Using the output surface means only the consequent fuzzy sets $G_{1}$ and $G_{2}$ are require definition as the antecedent sets $F_{1}$ and $F_{2}$ will not be used. Both of the consequent sets have triangular membership functions, the type-1 version of which are depicted in Figure 5. The remaining four systems will all be extended from the type- 1 system using $G_{1}$ and $G_{2}$. The type-reduced, direct defuzzifier and sampling defuzzifier systems will all use the interval type-2 fuzzy sets $\tilde{G}_{1}$ and $\tilde{G}_{2}$ depicted in Figure 6 . All the fuzzy sets will be discretised at 10 equally spaced points across their respective supports. Each secondary membership function of sampling system will be discretised at 10 equally spaced points across the support of that secondary membership function. The sampling defuzzifer will take a random sample of 30 embedded sets. We choose 30 as the sample size as this number should be large enough to give a normal distribution of results. The non-stationary fuzzy system that will take the final defuzzified value as the mean of the results from the 30 iterations. The non-stationary system will use a random perturbation function to alter the membership functions of the type-1 fuzzy sets $G_{1}$ and $G_{2}$. These perturbations will create membership functions that always fall within the bounds of the interval type-2 fuzzy sets that will be used as for comparative purposes.

An output surface plot will be drawn for each of the five systems using both the minimum and product t-norms. Previous research suggests that interval type- 1 systems should have smoother output surfaces than type-1 systems. We expect the non-stationary system to emulate to some extent the smoothness of the interval type- 2 systems. The sampling defuzzifier is an unknown quantity.

This section has give description of the fuzzy systems compared in this work. The use output surface diagrams has also be explored. The following section give the results from all the fuzzy systems.

\section{RESULTS}

In this section, diagrams of the output surfaces of the various fuzzy systems are given. Each of the systems will be compared under both the minimum and product t-norms. When assessing the output surface plots we are looking for two main criteria, smoothness of the surface, form or shape of the surface. A surface which has soft curving lines with no jagged edge means the system will have smooth control characteristics. Each of the lines represents an output value. Each of these essentially represents a function of the two input parameters, the firing levels of the two rules. The smoother these lines, the smoother the control performance. The shape of the surface defines the control performance. We are looking for similarities in the shapes and for inconsistencies between the plots.

\section{A. Systems Using Minimum t-norm}

The output surface plots of the five fuzzy systems using the minimum t-norm are given in following figures:

- Figure 7 depicts the output plot for the type-1 fuzzy system;

- Figure 8 depicts the output plot for the interval type-2 fuzzy system that uses type-reduction;

- Figure 9 depicts the output plot for the interval type-2 fuzzy system that uses direct defuzzifier;

- Figure 10 depicts the output plot for the interval type-2 fuzzy system that uses the sampling defuzzifier, and;

- Figure 11 depicts the output plot for the non-stationary fuzzy system.

\section{B. Systems Using Product t-norm}

The output surface plots of the five fuzzy systems using the product t-norm are given in following figures:

- Figure 12 depicts the output plot for the type-1 fuzzy system;

- Figure 13 depicts the output plot for the interval type-2 fuzzy system that uses type-reduction; 


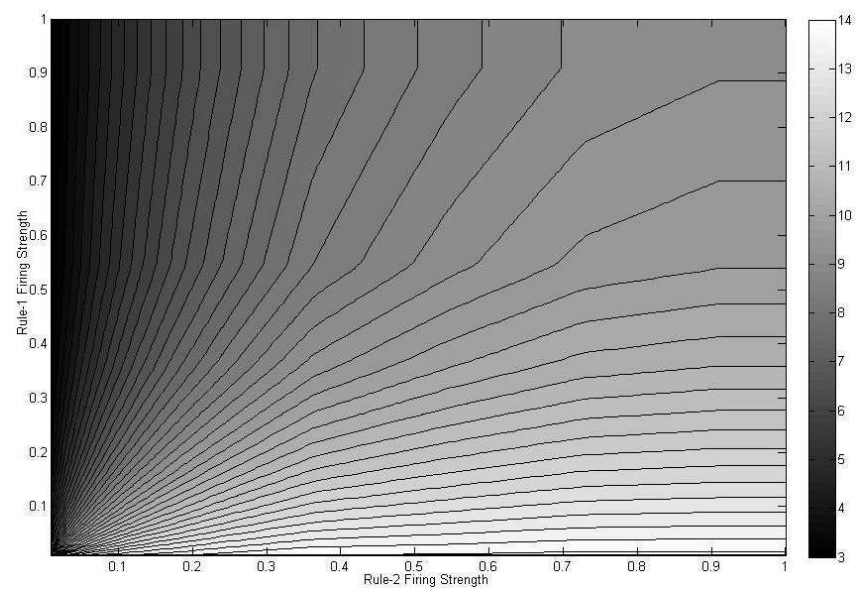

Fig. 7. Output Surface of the Type-1 System Using The Minimum T-norm.

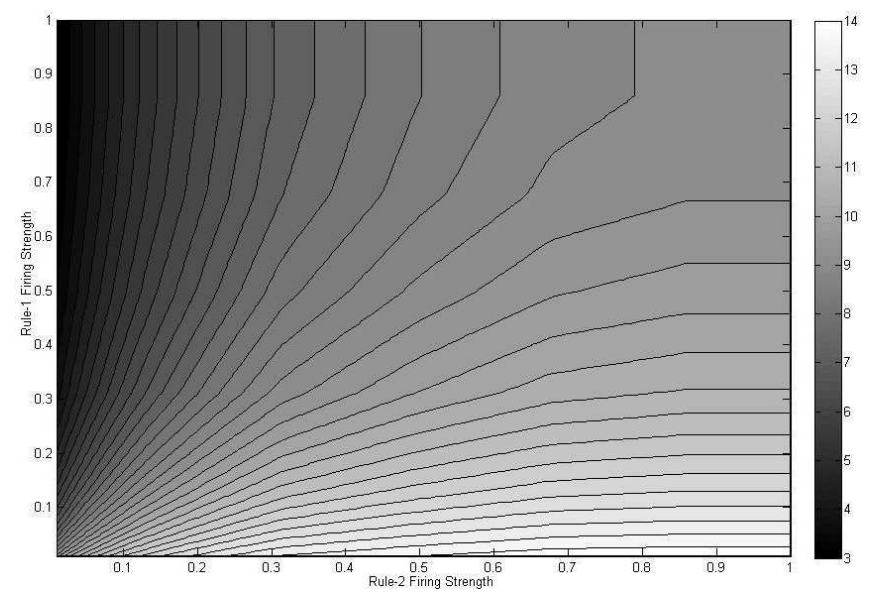

Fig. 8. Output Surface of the Type-Reduction System Using The Minimum T-norm.

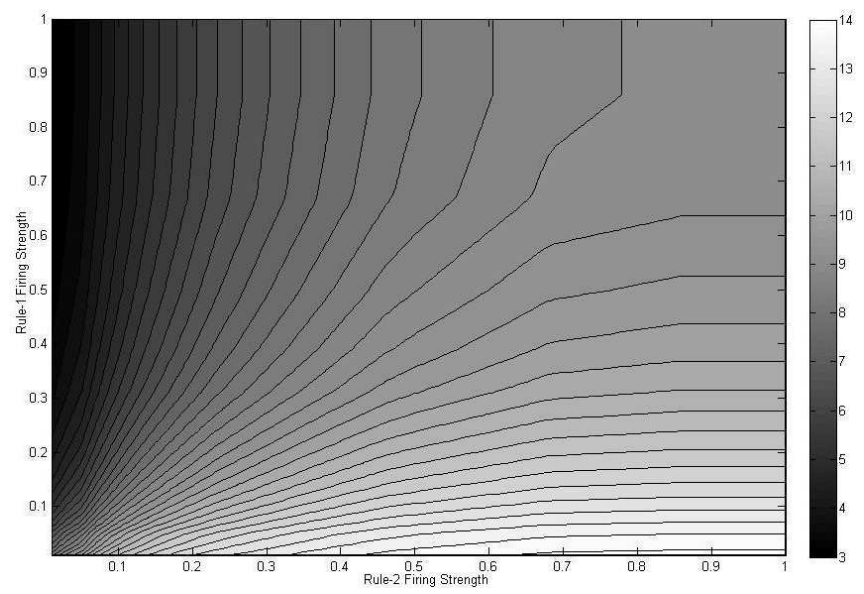

Fig. 9. Output Surface of the Direct Defuzzfier System Using The Minimum T-norm.

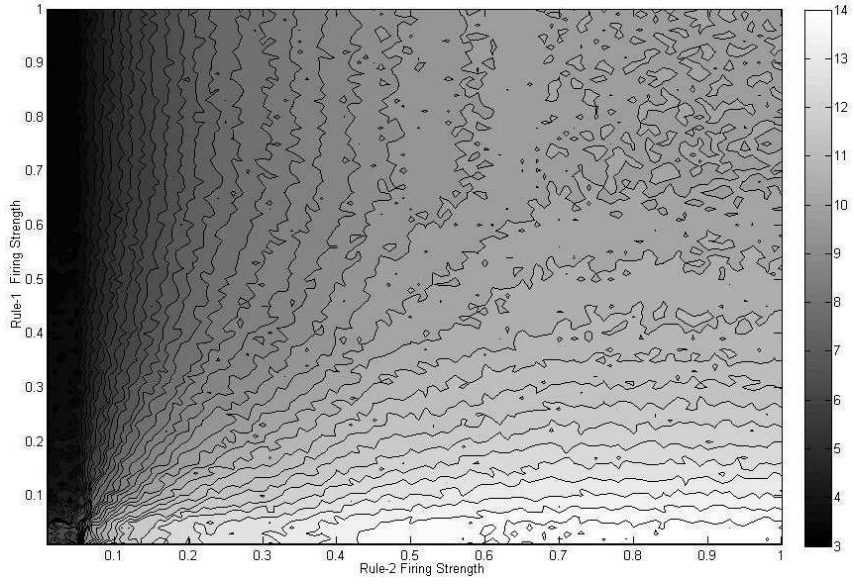

Fig. 10. Output Surface of the Sampling Defuzzifier System Using The Minimum T-norm.

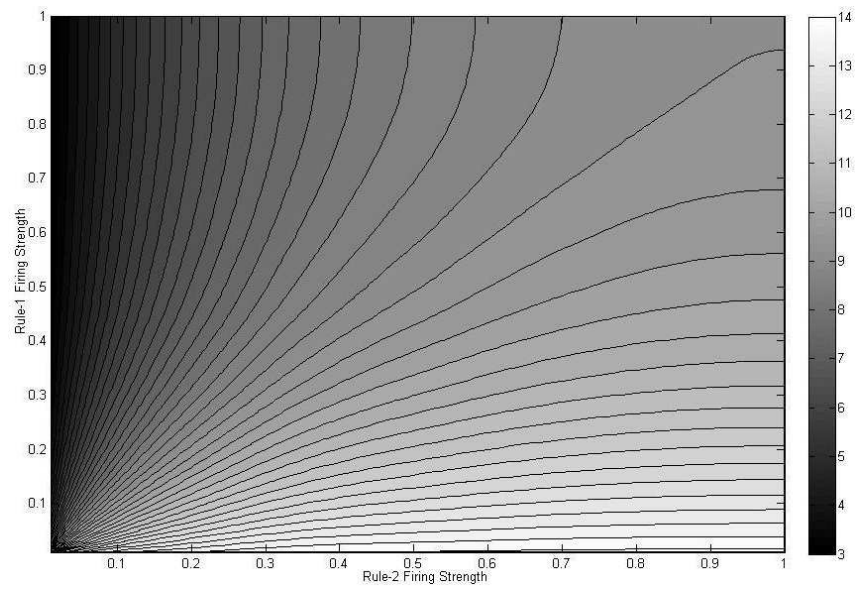

Fig. 11. Output Surface of the Non-Stationary Sampling System Using The Minimum T-norm.

- Figure 14 depicts the output plot for the interval type-2 fuzzy system that uses direct defuzzifier;

- Figure 15 depicts the output plot for the interval type-2 fuzzy system that uses the sampling defuzzifier, and;

- Figure 16 depicts the output plot for the non-stationary fuzzy system.

\section{DISCUSSION}

The previous section gave the various output surface plots. In this section we discuss what these plots can tell us about the fuzzy technologies that produced them.

\section{A. Systems Using Minimum t-norm}

It is immediately clear that the sampling defuzzifier stands out from the other plots. Although the form of the plot is similar to the others, the lines are quite distinct. The lines are extremely jagged with small closed patches portraying a noisy surface. At the other end of the spectrum the lines of the non-stationary system display a good deal more smoothness than the other systems. The curves are soft and 


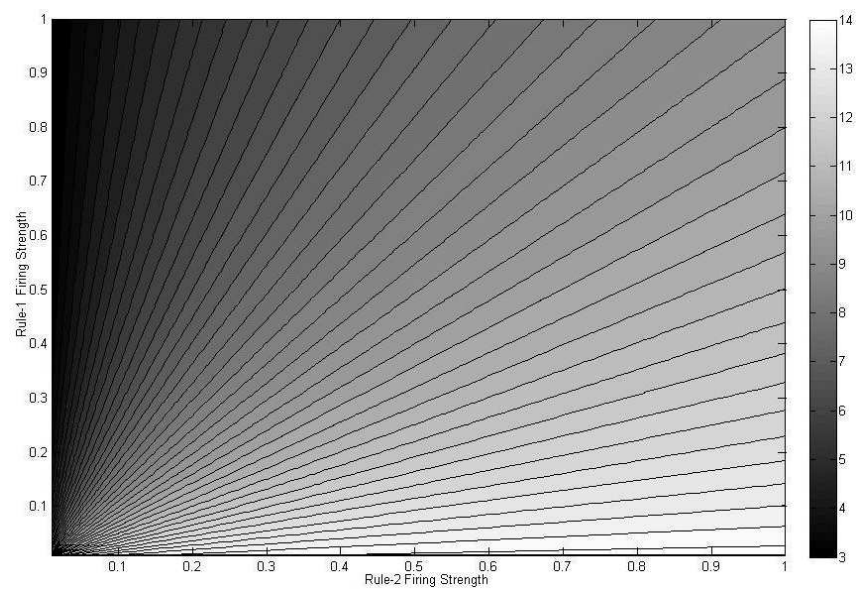

Fig. 12. Output Surface of the Type-1 System Using The Product T-norm.

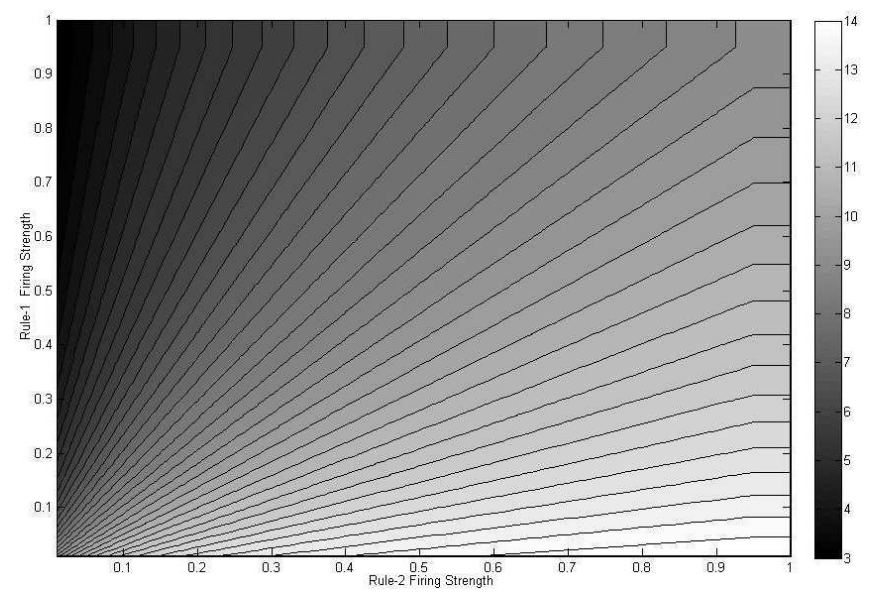

Fig. 13. Output Surface of the Type-Reduction System Using The Product T-norm.

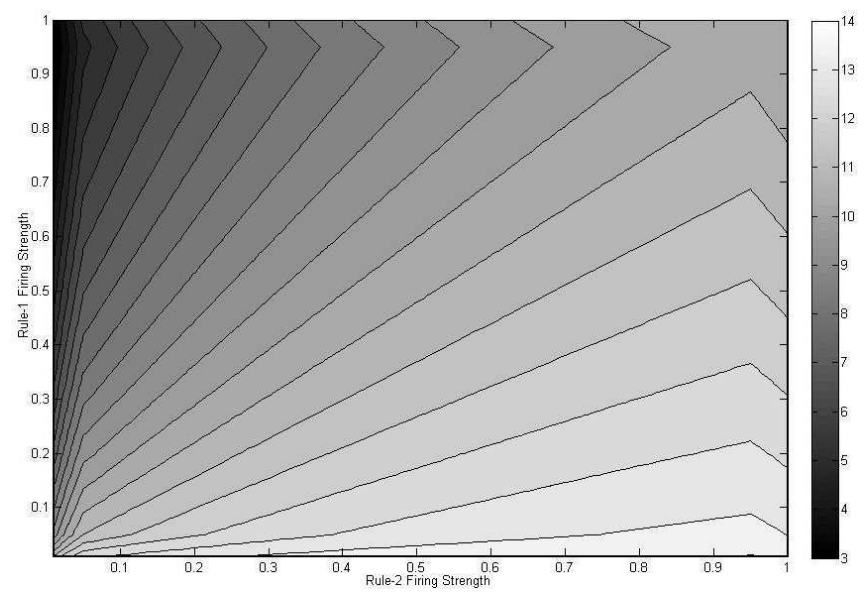

Fig. 14. Output Surface of the Direct Defuzzfier System Using The Product T-norm.

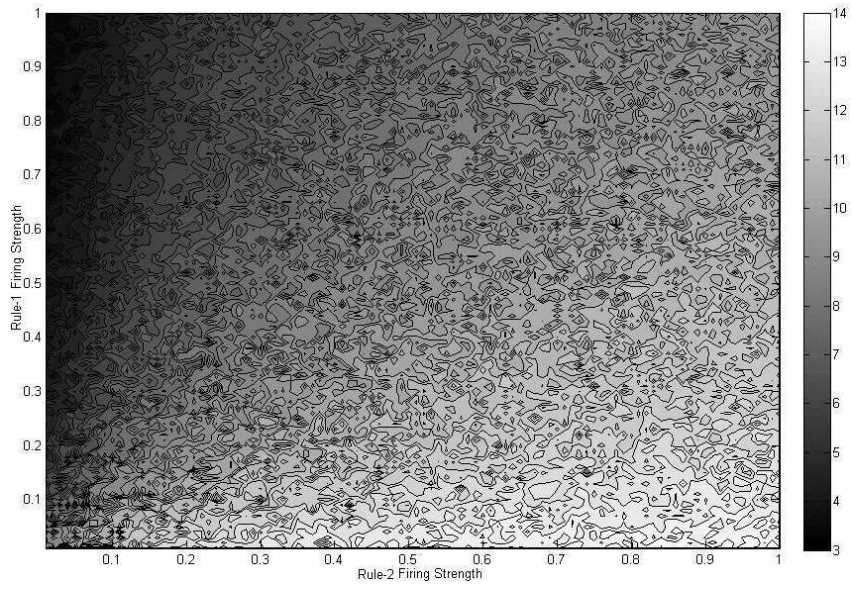

Fig. 15. Output Surface of the Sampling Defuzzifier System Using The Product T-norm

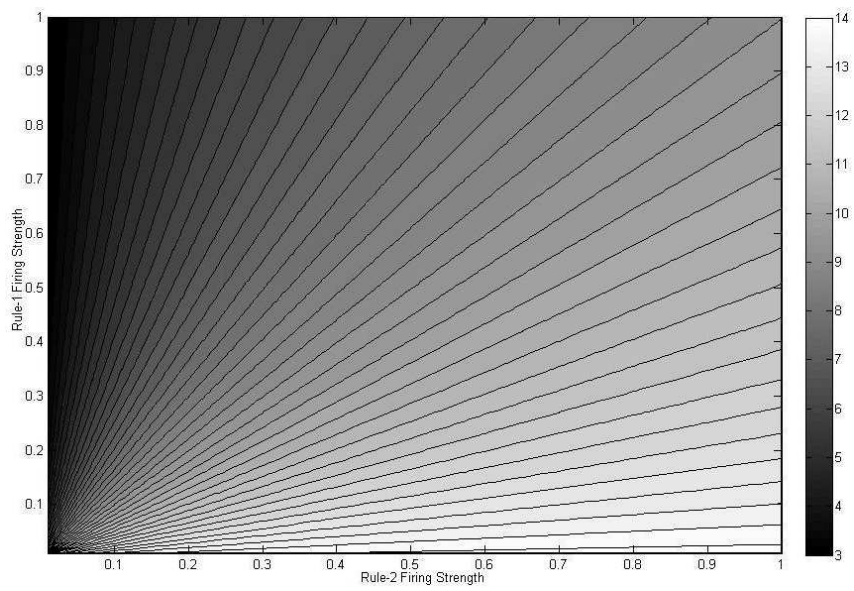

Fig. 16. Output Surface of the Non-Stationary Sampling System Using The Product T-norm.

sweeping with no jagged edges. This system clearly has a smooth control performance. The direct defuzzifier and the non-stationary system gave near identical performance. Small differences can be seen when both rule firing levels are less than 0.15 , but these are by no means significant discrepancies. The lines in these plots are reasonably smooth, with some abrupt linear sections where the rule firing level are around $0.7-0.8$. The shape of these surfaces are different to the type- 1 surface. The type-1 surface shows more compact lines around the bottom-left to top-right diagonal area, representing a sharper surface gradient. The shape of the non-stationary surface appears to be somewhere in between the two. The lines of the type-1 surface contain larger abrupt linear portions that the type-reducer and direct defuzzifier systems. The type- 1 lines are also show less smoothness with a second section of linear portions when the rule firing level are between 0.4 and 0.6 . The type- 1 would clearly give a less smooth control performance compared to the type-reducer and direct defuzzifier systems. 


\section{B. Systems Using Product t-norm}

The systems exhibited different characteristics under the product t-norm compared with the minimum t-norm. Again the sampling defuzzifier gave very noisy surface compared to the other systems, although its shape broadly matched the other surfaces. The type-1 and non-stationary systems gave near identical surfaces. Both had completely linear functions with similar distances between the lines. The type-reducer gave a very similar surface to the type- 1 and non-stationary systems. There are some discrepancies at the edges of the surface. The type-reducer shows lines emanating from the axis up to values of 0.6 , the other two these stop at around 0.4. The type-reducer also has small perpendicular linear portions at rule firing values of around 0.95 . The direct defuzzifier gave quite a different performance. The distances between the lines are much greater. Generally the output values on the surface are mush lower. The lines display strange characteristics when the rule-1 firing level is less than 0.5. The short perpendicular sections displayed by the typereducer are accentuated in the direct defuzzifier. At values of 0.95 the lines reverse back at angles of around $90^{\circ}$.

Under the product t-norm the type-1, type-reducer and non-stationary systems all performed well. The sampling defuzzifier again suffered from high noise levels. The direct defuzzifier could not match the performance of the typereducing system and display some strange characteristics.

This section has discussed the features and characteristics of the various systems. The final section draws this work to a conclusion.

\section{CONCLUSION}

This paper has defined the novel direct defuzzifier for interval type-2 fuzzy logic systems. The first application of the sampling defuzzifier to interval systems has been given. We have compared the performance of these fuzzy systems to type-1, interval type-2 using type-reduction and non-stationary fuzzy systems.

We found the sampling method gave a noisy performance. Increasing the number of samples and increasing the level of discretisation may reduce, even eliminate this noise. We suggest future work should explore this possibility.

The direct defuzzifier performed very well under the minimum t-norm, matching the type-reduction systems performance. Under the product t-norm the direct defuzzifier showed some strange characteristics. These differences should be further explored. It may be that this defuzzifier is only suitable for certain systems.

The surprising result in this experiment was the performance of the non-stationary fuzzy system. We expected this system to give a similar performance to the type-1 system. We observed that under the minimum t-norm the non-stationary system gave a much smoother surface than any of the other systems. Under the product t-norm the nonstationary system was as good as any of the other systems.

In this paper we have seen a possible alternative to typereduction in the direct defuzzifier. We have shown that the sampling defuzzifier may not be suitable for interval systems, although more work is certainly needed. The most import result in this paper is the performance of the nonstationary fuzzy system. We have seen the non-stationary system outperform the other fuzzy technologies. This result raises important questions about the direction of type- 2 fuzzy research and about how engineers should model uncertainty in practical applications.

\section{REFERENCES}

[1] S. Coupland and R. John. A New and Efficient Method for the Type2 Meet Operation. In Proc. FUZZ-IEEE 2004, pages 959 - 964, Budapest, Hungary, July 2004.

[2] S. Coupland and R. John. Fuzzy Logic and Computational Geometry. In Proc. RASC 2004, pages 3 - 8, Nottingham, England, December 2004.

[3] S. Coupland and R. John. Geometric Interval Type-2 Fuzzy Systems. In Proc. EUSFLAT05, pages 449 - 454, Bacelona, Spain, September 2005.

[4] S. Coupland and R. John. Towards More Efficient Type-2 Fuzzy Logic Systems. In Proc. FUZZ-IEEE05, pages 236 - 241, Reno, NV, USA, May 2005.

[5] J. Figueroa, J. Posada, J. Soriano, M. Melgarejo, and S. Rojas. A Type-2 Fuzzy Controller for Tracking Mobile Objects in the Context of Robotic Soccer Games. In Proc. FUZZ-IEEE05, pages 359 - 364, Reno, AZ, USA, May 2005.

[6] J. M. Garibaldi, S. Musikasuwan, and T. Ozen. The Association between Non-Stationary and Interval Type-2 Fuzzy Sets: A Case Study. In Proc. FUZZ-IEEE 2005, pages 224-229, Reno, NV, USA, May 2005.

[7] S. Greenfield, R. John, and S. Coupland. A Novel Sampling Method for Type-2 Defuzzification. In Proc. UKCI 05, 2005.

[8] H. Hagras. A Hierarchical Type-2 Fuzzy Logic Control Architecture for Autonomous Mobile Robots. IEEE Trans. Fuzzy Systems, 12:524539, 2004.

[9] N. N. Karnik and J. M. Mendel. Type-2 Fuzzy Logic Systems: TypeReduction. In Proceedings IEEE Systems, Man and Cybernetics, pages 2046-2051, 1998

[10] N. N. Karnik and J. M. Mendel. Centroid of a type-2 fuzzy Set. Information Sciences, 132:195-220, 2001.

[11] C. Lynch, H. Hagras, and V. Callaghan. Embedded Type-2 FLC for Real-Time Speed Control of Marine and Traction Diesel Engines. In Proc. FUZZ-IEEE05, pages 347 - 352, Reno, AZ, USA, May 2005.

[12] J. M. Mendel. Uncertain Rule-Based Fuzzy Logic Systems: Introduction and New Directions. Prentice-Hall, Upper Saddle River, NJ, 2001.

[13] J. M. Mendel and R. I. John. Type-2 Fuzzy Sets Made Simple. IEEE Transaction on Fuzzy Systems, 10(2):117-127, 2002.

[14] T. Ozen and J. M. Garibaldi. Effect of Type-2 Fuzzy Membership Function Shape on Modelling Variation in Human Decision Making. In Proc. FUZZ-IEEE 2004, pages 971 - 976, Budapest, Hungary, July 2004.

[15] D. Wu and W. W. Tan. A Type-2 Fuzzy Logic Controller for the Liquid-level Process. In Proc. FUZZ-IEEE 2004, pages 953 - 958, Budapest, Hungary, July 2004.

[16] H. Wu and J. M. Mendel. Uncertainty bounds and their use in the design of interval type-2 fuzzy logic systems. IEEE Transactions on Fuzzy Systems, pages 622-639, October 2002. 